$$
\begin{aligned}
& \bar{V}_{g} \quad=\text { mean volumetric gas flow rate into chamber }\left[\mathrm{m}^{3} / \mathrm{s}\right] \\
& \text { We } \quad=\text { Weber number }=\rho_{t} U_{g}^{2} d_{o} / \sigma \\
& \begin{array}{ll}
\Delta \rho & =\rho_{l}-\rho_{g} \\
\mu_{l} & =\text { viscosity of liquid }
\end{array} \\
& \rho_{g}, \rho_{l} \quad=\text { density of gas and liquid } \\
& \sigma \quad=\text { surface tension }
\end{aligned}
$$

\section{Literature Cited}

1) Akagi, Y. and T. Takahashi: Kagaku Kogaku Ronbunshu, 9, 594 (1983).
2) Akagi, Y,, M. Nishikaze, S. Yamamoto and T. Takahashi: Kagaku Kogaku Ronbunshu, 7, 442 (1981).

3) Kumar, R. and N. R. Kuloor: "Advances in Chemical Engineering," 8, 255 (1970).

4) McCann, D. J. and R. G. H. Prince: Chem. Eng. Sci., 24, 801 (1969).

5) Miyahara, T., N. Haga and T. Takahashi: Intern. Chem. Eng., 24, 524 (1983).

6) Tadaki, T. and S. Maeda: Kagaku Kōgaku, 27, 147 (1963).

7) Zenze, F. A. L., L. Stone and M. Crane: Hydrocarbon Processing, 46, 138 (1967).

\title{
BUBBLE VOLUME IN SINGLE BUBBLING REGIME WITH WEEPING AT A SUBMERGED ORIFICE
}

\author{
TOSHIRO MIYAHARA AND TERUO TAKAHASHI \\ Department of Industrial Chemistry, Okayama University, Okayama 700
}

Key Words: Fluid Mechanics, Bubble Volume, Weeping, Single Orifice, Two-Stage Model

\begin{abstract}
Bubble volumes in the single bubbling regime, which is one of bubble formation regimes with weeping, are larger than those for bubble formation without weeping. A theoretical treatment for bubble formation with weeping is performed to predict bubble volume in the single bubbling regime where weeping through an orifice occurs after the detachment of a bubble, and a comparison with measurements is made.
\end{abstract}

\section{Introduction}

A number of gas-liquid contacting devices in the chemical industry contain perforated plates because of their simplicity. Recently, industrial operation requires plates having relatively large free areas, which correspond to large hole diameters, where weeping occurs. Therefore, knowledge of bubble formation with weeping is important for the design or operation of gas-liquid contacting devices with perforated plates.

To clarify the gas-liquid contacting mechanism, many investigators ${ }^{3-5,8,11,14,17,18)}$ have studied bubble formation at single holes. Less attention, however, has been given to bubble formation phenomena with weeping.

McCann et al. ${ }^{7)}$ have proposed a model to describe bubble volume and weeping rate theoretically using potential flow analysis, but they did not consider the effect of viscosity.

In a previous paper, ${ }^{9)}$ the authors observed four regimes of bubble formation with weeping based on operating conditions and orifice geometries: single

\footnotetext{
Received March 23, 1984. Correspondence concerning this article should be addressed
} to T. Miyahara. bubbling, doubling, pairing and jetting.

In the present paper, a theoretical treatment for bubble formation with weeping is given by using the previous two-stage model and is shown to be in fairly good agreement with measurements in the single bubble formation regime, which is one of the bubble formation regimes with weeping observed previously by the authors. ${ }^{9)}$

\section{Experimental}

The experimental apparatus and procedure used are the same as those described in the previous paper. ${ }^{9)}$

All of the orifices used with diameters of $3-13.2 \mathrm{~mm}$ were made of brass plate of $1 \mathrm{~mm}$ thickness $\left(p_{t} / d_{o}=\right.$ $0.075-0.33$ ). Eight different liquids were studied: water, aqueous ethanol solutions, aqueous millet jelly solutions and aqueous glycerine solution. Experiments were carried out over a range of densities of $953-1263 \mathrm{~kg} / \mathrm{m}^{3}$, a range of viscosities of $1 \times 10^{-3}$ $13.5 \times 10^{-3} \mathrm{~Pa} \cdot \mathrm{s}$ and a range of surface tensions of $35.1 \times 10^{-3}-72.0 \times 10^{-3} \mathrm{~N} / \mathrm{m}$. The details are shown in Table 1, which is the same as that in the previous paper. ${ }^{9)}$ Chamber volumes changed from 75 to $14,000 \mathrm{~cm}^{3}$. 
Table 1. Physical properties of liquids employed

\begin{tabular}{lrlll}
\hline \multicolumn{1}{c}{ Liquid } & $\rho_{I}\left[\mathrm{~kg} / \mathrm{m}^{3}\right]$ & $\mu_{l}[\mathrm{~Pa} \cdot \mathrm{s}]$ & $\sigma[\mathrm{N} / \mathrm{m}]$ \\
\hline Water & 1000 & 0.001 & $72.0 \times 10^{-3}$ \\
Aqueous ethanol soln. 1 & 969 & 0.00255 & $37.2 \times 10^{-3}$ \\
Aqueous ethanol soln. 2 & 953 & 0.00261 & $35.6 \times 10^{-3}$ \\
Aqueous ethanol soln. 3 & 964 & 0.00271 & $35.1 \times 10^{-3}$ \\
Aqueous millet jelly soln. 1 & 1263 & 0.0410 & $70.7 \times 10^{-3}$ \\
Aqueous millet jelly soln. 2 & 1257 & 0.0353 & $71.4 \times 10^{-3}$ \\
Aqueous millet jelly soln. 3 & 1308 & 0.135 & $66.5 \times 10^{-3}$ \\
Aqueous glycerine soln. & 1196 & 0.0356 & $60.8 \times 10^{-3}$ \\
\hline
\end{tabular}

By assuming that bubbles were all of the same size, the volumes were obtained as the amount of volumetric gas flow rate divided by the frequency.

\section{Results and Discussion}

\subsection{Bubble volume in single bubbling regime}

Figures 1, 2 and 3 show bubble volumes in the single bubbling regime described previously, ${ }^{9)}$ for various liquids, orifice diameters and chamber numbers. Bubble volumes increase with increasing chamber volumes at any gas flow rate. However, the effect of chamber volume is especially apparent at low gas flow rates when bubble volume is approximately constant. These trends are similar to those in single bubble formation without weeping at an orifice with small hole diameter. This was previously shown by the authors, ${ }^{16)}$ who have derived a correlating equation for the volume of a bubble formed at a single orifice by assuming that bubble volumes in each of the regions of low and high gas flow rates are additive. In each figure, the calculated curves from the correlating equation by the authors ${ }^{16)}$ are shown by dashed lines. The measurements are not so well correlated with the previous results by the authors. ${ }^{16)}$ Moreover, the calculated values are smaller than the measurements in all of the regions of gas flow rate and chamber volume.

According to Tadaki et al., ${ }^{14)}$ approximately constant bubble volumes without weeping at low gas flow rates are expressed as

$$
\rho_{1} g V_{b}=C_{c} \pi d_{o} \sigma
$$

where $C_{c}$ is a function of the chamber number $N_{c}$.

Figure 4 shows the coefficient $C_{c}$ in Eq. (1). The solid line is the result by Tadaki et al.., ${ }^{14)}$ who studied the bubble formation without weeping. As can be seen from this figure, $C_{c}$ is a few times larger than that of Tadaki et al. ${ }^{14)}$ in all regions of $N_{c}$ : that is, the volume of a bubble with weeping formed at an orifice is rather larger than that without weeping.

These differences are perhaps due to pressure fluctuation in the gas chamber or liquid weeping. Therefore, we need to propose an improved model of bubble formation which explains the measurements

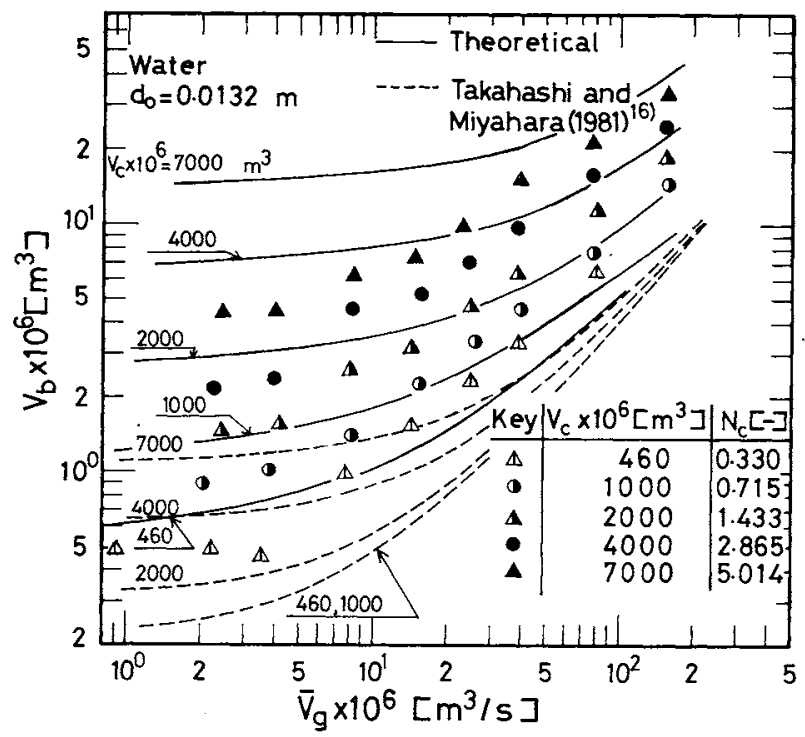

Fig. 1. Bubble volume in single bubbling regime.

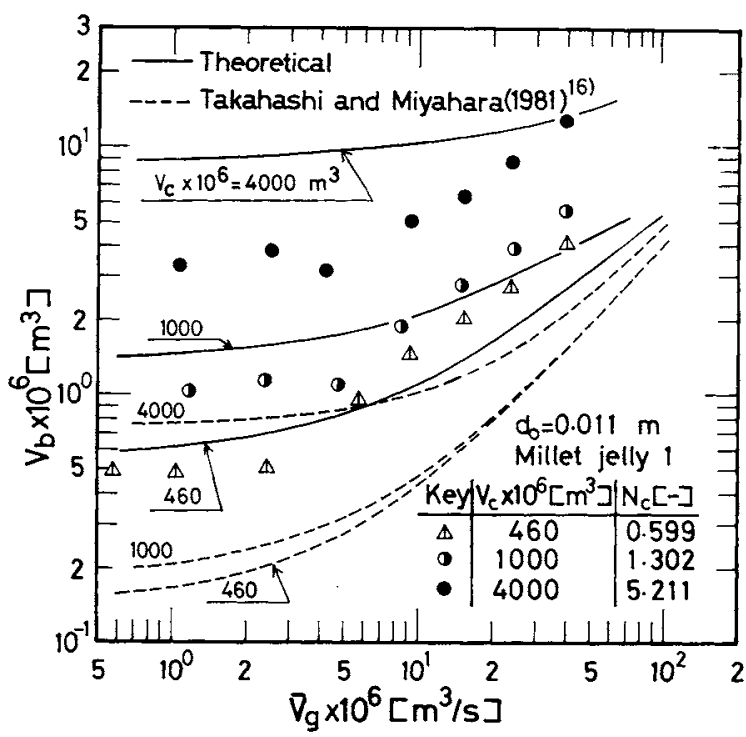

Fig. 2. Bubble volume in single bubbling regime

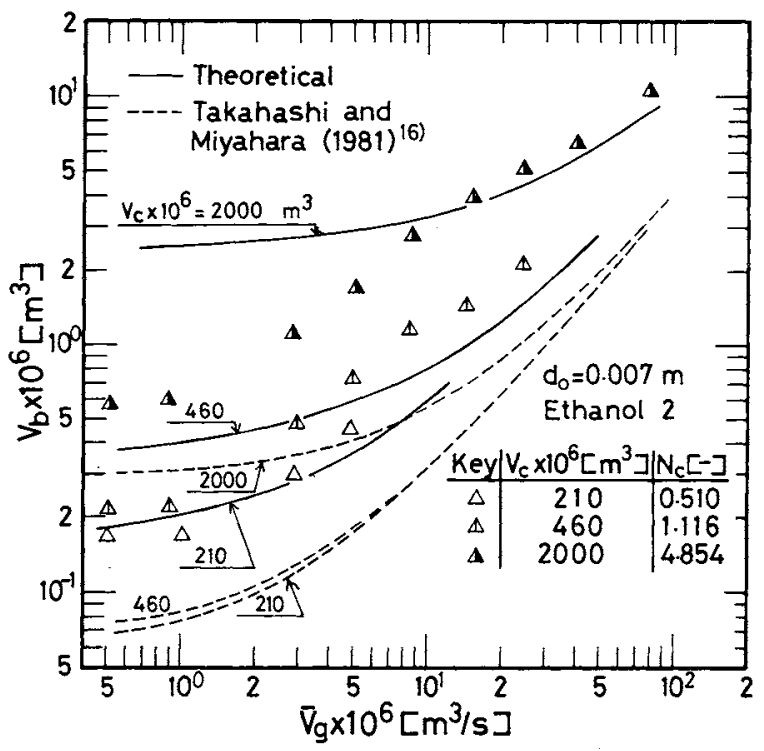

Fig. 3. Bubble volume in single bubbling regime. 


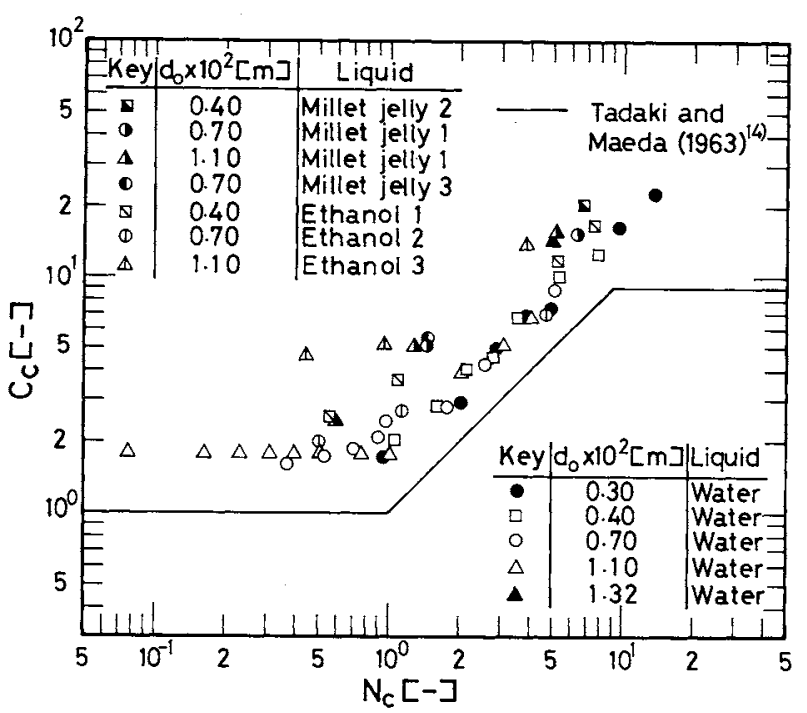

Fig. 4. Comparison between bubble volumes with and without weeping in the range of constant bubble volume.

well.

\subsection{Theoretical consideration}

As has been already described, ${ }^{9}$ ) in the single bubbling regime the bubbles are almost all of the same size and weeping does not occur during bubble formation, whereas it does occur after the detachment of a bubble. Further, initially an almost spherical bubble is formed at an orifice, proceeds to grow a neck and then detaches. That is, the growth of the bubble is a two-stage process as in Fig. 5, which shows that the previous two-stage model ${ }^{5,15,16)}$ is valid.

Assuming that gas flow rate from the chamber into a bubble is dependent on time, the termination of the first stage when the bubble is assumed to stay at the orifice is

$$
\begin{aligned}
& \frac{\left(\rho_{g}+\frac{11}{16} \rho_{l}\right)}{12 \pi\left\{3 V_{b} /(4 \pi)\right\}^{2 / 3}}\left(3 V_{b} \frac{d V_{g}}{d t}+V_{g}^{2}\right)+\pi d_{o} \sigma-\left(\rho_{l}-\rho_{g}\right) g V_{b} \\
& -\frac{4 \rho_{g} V_{g}^{2}}{\pi d_{o}^{2}}+C_{D}(\pi / 8)\left(6 V_{b} / \pi\right)^{2 / 3} \frac{\rho_{l} V_{g}^{2}}{\left[4 \pi\left\{3 V_{b} /(4 \pi)\right\}^{2 / 3}\right]^{2}}=0
\end{aligned}
$$

The fourth term on the left-hand side of this equation is the force due to the momentum of the gases. The momentum term is involved due to the assumption that the instantaneous gas flow rate through a hole is fairly large because of the pressure fluctuation in the gas chamber. The equation of motion for the second stage is written as follows:

$$
\begin{aligned}
& \left(\rho_{g}+\frac{11}{16} \rho_{l}\right) V_{b} \frac{d v_{n}}{d t}+\left(\rho_{g}+\frac{11}{16} \rho_{l}\right) V_{g} v_{n}+\frac{\left(\rho_{g}+\frac{11}{16} \rho_{l}\right)}{12 \pi\left\{3 V_{b} /(4 \pi)\right\}^{2 / 3}} \\
& \times\left(3 V_{b} \frac{d V_{g}}{d t}+V_{g}^{2}\right)=\left(\rho_{l}-\rho_{g}\right) g V_{b}-N \pi d_{o} \sigma+\frac{4 \rho_{g} V_{g}^{2}}{\pi d_{o}^{2}} \\
& -C_{D}(\pi / 8) d_{b}^{2} \rho_{l}\left(v+v_{n}\right)^{2}
\end{aligned}
$$

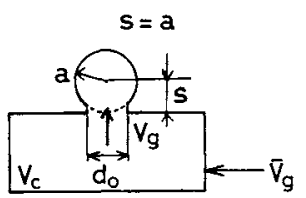

First stage

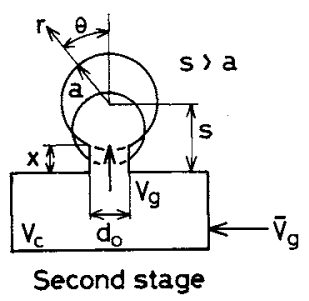

Fig. 5. A model for bubble formation with weeping.

where

$$
v=\frac{d a}{d t}
$$

The coefficient $N$ involved in Eq. (3) is a correction factor based on the contact condition already defined by the authors, ${ }^{15)}$ who have reported $N=1 / 2$. The growing velocity of the neck is

$$
\frac{d x}{d t}=v_{n}
$$

The pressure fluctuation is given by the following equation ${ }^{6)}$ :

$$
\frac{d P_{c}}{d t}=\frac{\gamma P_{c}}{V_{c}}\left(\bar{V}_{g}-4 \pi a^{2} \frac{d a}{d t}\right)
$$

$\gamma$ is the polytropic coefficient, being equal to the specific heat ratio for adiabatic behavior; in a normal diatomic gas (air being classed such a gas), $\gamma=1.41$.

McCann et al. ${ }^{7)}$ have derived the theoretical pressure inside a bubble for $s \geqq a$ using potential flow analysis for a rising, expanding bubble as follows:

$$
\begin{aligned}
P_{b} & =\rho_{l}\left\{\left(\frac{3}{2}\right)\left(\frac{d a}{d t}\right)^{2}+a \frac{d^{2} a}{d t^{2}}-g s\right\}-\frac{\rho_{l}}{4}\left(\frac{d s}{d t}\right)^{2}+\frac{2 \sigma}{a}+P_{\infty} \\
& =P_{s}+P_{\infty}+\frac{2 \sigma}{a}
\end{aligned}
$$

where

$$
P_{\infty}=P_{o}+\rho_{1} g h .
$$

However, Eq. (7) is valid only for an ideal fluid. It is difficult to derive theoretically the concrete form of $P_{b}$ for a real liquid. Therefore, we will try to involve the force due to viscosity.

Poritsky ${ }^{12)}$ was the first to illustrate the effect of viscosity from the stress conditions on the surface of a spherical bubble for an incompressible fluid under the assumption that the bubble is a spherical symmetry of the motion, which is not true in practice. Hence we obtain for the rising, expanding bubble using a similar analysis to that of Poritsky ${ }^{12)}$ (See Appendix):

$$
P_{b}-P_{s}=P_{\infty}+\frac{2 \sigma}{a}+\frac{4 \mu_{l}}{a} \frac{d a}{d t}
$$

Substitution of Eq. (7) into Eq. (8) yields 


$$
\begin{aligned}
P_{b}= & \rho_{l}\left\{\left(\frac{3}{2}\right)\left(\frac{d a}{d t}\right)^{2}+a \frac{d^{2} a}{d t^{2}}-g a\right\} \\
& -\frac{\rho_{l} v^{2}}{4}+\frac{2 \sigma}{a}+P_{\infty}+\frac{4 \mu_{l}}{a} \frac{d a}{d t}
\end{aligned}
$$

for the first stage

and

$$
\begin{aligned}
P_{b}= & \rho_{l}\left\{\left(\frac{3}{2}\right)\left(\frac{d a}{d t}\right)^{2}+a \frac{d^{2} a}{d t^{2}}-g s\right\} \\
& -\frac{\rho_{l} v^{2}}{4}+\frac{2 \sigma}{a}+P_{\infty}+\frac{4 \mu_{l}}{a} \frac{d a}{d t}
\end{aligned}
$$

for the second stage.

Similar analyses reflecting the viscous contribution have been carried out by Guy et al. ${ }^{11}$ and Pinczewski. ${ }^{11)}$

The flow rate of gas $V_{g}$ into a bubble is expressed as

$$
V_{g}=\frac{d V_{b}}{d t}=4 \pi a^{2} \frac{d a}{d t}=k(\Delta P)^{0.5}
$$

where

$$
\begin{gathered}
\Delta P=P_{c}-P_{b}, k=(\pi / 4) d_{o}^{2} \sqrt{2 /\left(\rho_{g} C_{g}\right)} \\
\text { and } C_{g}=1.5+4 p_{t} f / d_{o} .
\end{gathered}
$$

The substitution of Eq. (11) into Eqs. (9) and (10) gives, after some manipulation,

$$
\begin{aligned}
\frac{d^{2} a}{d t^{2}}= & \frac{1}{\rho_{l} a}\left(P_{c}-P_{\infty}-\frac{2 \sigma}{a}+\rho_{l} g a\right) \\
& +\left(\frac{1}{4 a}-\frac{3}{2 a}-\frac{16 \pi^{2} a^{3}}{\rho_{l} k^{2}}\right)\left(\frac{d a}{d t}\right)^{2}-\frac{4 \mu_{l}}{\rho_{l} a^{2}} \frac{d a}{d t}
\end{aligned}
$$

for the first stage

and

$$
\begin{aligned}
\frac{d^{2} a}{d t^{2}}= & \frac{1}{\rho_{l} a}\left(P_{c}-P_{\infty}-\frac{2 \sigma}{a}+\rho_{l} g a+\rho_{l} g x\right)+\frac{1}{4 a}\left(\frac{d x}{d t}+\frac{d a}{d t}\right)^{2} \\
& -\frac{4 \mu_{l}}{\rho_{l} a^{2}} \frac{d a}{d t}-\left(\frac{16 \pi^{2} a^{3}}{\rho_{l} k^{2}}+\frac{3}{2 a}\right)\left(\frac{d a}{d t}\right)^{2}
\end{aligned}
$$

for the second stage.

The equations for the first stage, Eqs. (4), (6) and (12) can be solved simultaneously under the initial conditions $P_{c}=P_{o}+\rho_{1} g h+4 \sigma / d_{o}, \quad d a / d t=0$ and $a=d_{o} / 2$. The termination of the first stage is determined by Eq. (2). The equations for the second stage, Eqs. (3), (4), (5), (6) and (13) can be solved simultaneously under the initial conditions

$P_{\mathrm{c}}=\left(P_{\mathrm{c}}\right)_{f}, \quad \frac{d a}{d t}=\left(\frac{d a}{d t}\right)_{f}, \quad a=a_{f}, \quad v_{n}=0 \quad$ and $\quad x=0$

and the boundary condition

$$
x=a_{f} \quad \text { or } \quad \frac{d a}{d t}=0
$$

The former has been assumed by Kumar et al. ${ }^{5)}$ and the latter means the halting of the growth rate of a bubble.

We will use the expression derived by Hadamard ${ }^{2)}$ and Rybczynski ${ }^{13)}$ as the drag coefficient $C_{D}$, namely,

$$
C_{D}=\frac{16}{R e}
$$

The solid lines shown in Figs. 1, 2 and 3 are the results of calculation using the model mentioned above. The calculated curves fit roughly with the measurements over a wide range of experimental conditions. But the calculated values are larger than the experimental ones at small gas flow rates in large chamber volumes. On the other hand, when gas flow rates become large, the calculated values fit well with the measurements. The most likely reason for the discrepancy is presumably that at large chamber volumes bubbles become large and non-spherical in shape, and inertial force is dominant as compared with forces based on bubble shape at large gas flow rates.

\subsection{Comparison with other investigators' findings}

The pressure fluctuation in the gas chamber as a function of time during bubble formation which is supposed to be accompanied by weeping has been measured only by Kupferberg et al., ${ }^{6}{ }^{6}$ We show a comparison of the present theory with their experimental results in Fig. 6. As can be seen from this figure, the agreement between theory and experiment is generally good. We have found no reports concerned with bubble volumes for single bubble formation with weeping.

We have now shown how it is roughly possible to predict bubble volume with weeping for orifices with relatively large hole diameters.

\section{Conclusion}

Bubble volume with weeping at a single orifice was studied experimentally in the single bubbling regime. A theoretical development is proposed to predict the bubble volume. The following results were obtained:

1) The volume of a bubble with weeping formed at an orifice is rather larger than that without weeping.

2) A theoretical development for bubble formation which takes into account the effect of viscosity is proposed by using the previous two-stage model. Agreement with the experiments is roughly good under conditions for which the model is described, i.e., for the single bubbling regime.

\section{Appendix}

Derivation of Eq. (8)

At any point, the radial component of radial stress is related by means of the equation 


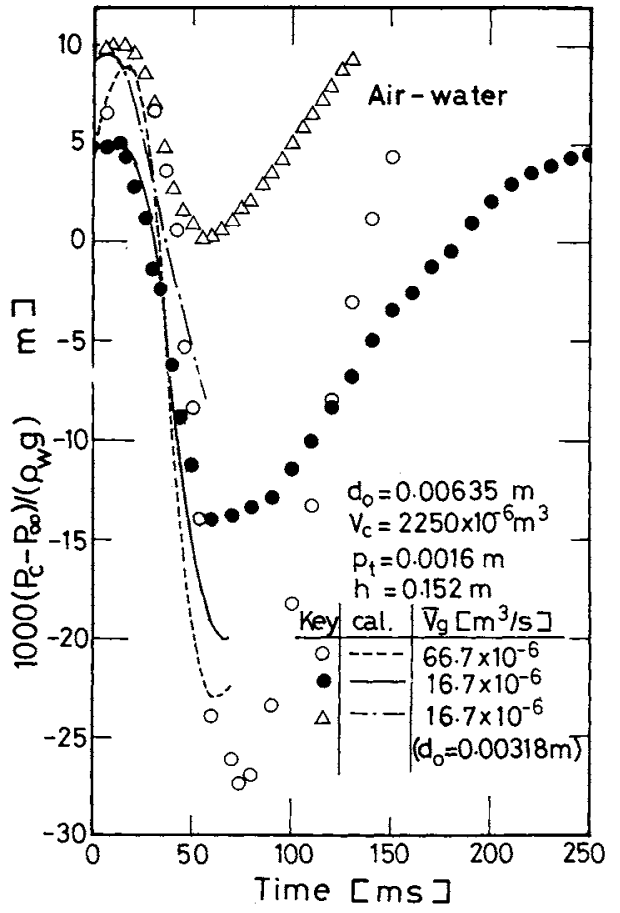

Fig. 6. Comparison with other investigators' findings.

$$
\tau_{r r}=\frac{2}{3}-\mu_{l}(\nabla \cdot v)-2 \mu_{l} \frac{\partial v_{r}}{\partial r}
$$

Since the fluid is incompressible the equation

$$
\nabla \cdot v=0
$$

holds. Therefore Eq. (A-1) can be expressed as follows:

$$
\tau_{r r}=-2 \mu_{l} \frac{\partial v_{r}}{\partial r}
$$

Now if for a viscous liquid $v_{r}$ is assumed to be expressed as the sum of the velocity due to the expanding motion and the velocity associated with the rising motion in infinite extent using the geometric configuration shown in Fig. A-1, then

$$
v_{r}=\frac{3}{2} U\left(\frac{a}{r}-\frac{1}{3} \frac{a^{3}}{r^{3}}\right) \cos \theta+\frac{a^{2}}{r^{2}} \frac{d a}{d t}
$$

Substitution of Eq. (A-4) into Eq. (A-3) gives the radial component of radial stress at the bubble surface within the liquid:

$$
\tau_{r r}=\frac{4 \mu_{l}}{a} \frac{d a}{d t}
$$

Therefore, we will try to include the radial component of radial stress, $\tau_{r r}$, in Eq. (7). It follows that

$$
P_{b}-P_{\mathrm{s}}=P_{\infty}+\frac{2 \sigma}{a}+\frac{4 \mu_{l}}{a} \frac{d a}{d t}
$$

\section{Acknowledgment}

The authors wish to thank Dr. R. D. LaNauze, who is associated with Division of Fossil Fuel, Delhi Road, North Ryde, NSW, Australia, for his useful comments on this subject, and Mr. Minoru Iwata for his assistance in the experimental measurements.

\section{Nomenclature}

$C_{6}$

$=$ bubble radius

$=$ constant defined by Eq. (1)

[m]

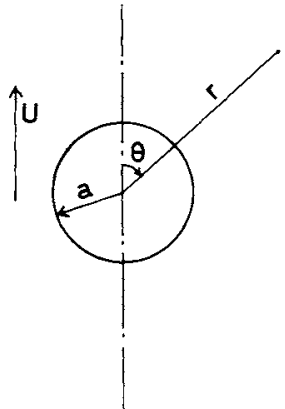

Fig. A-1. Geometric configuration of an expanding and rising bubble in still liquid.

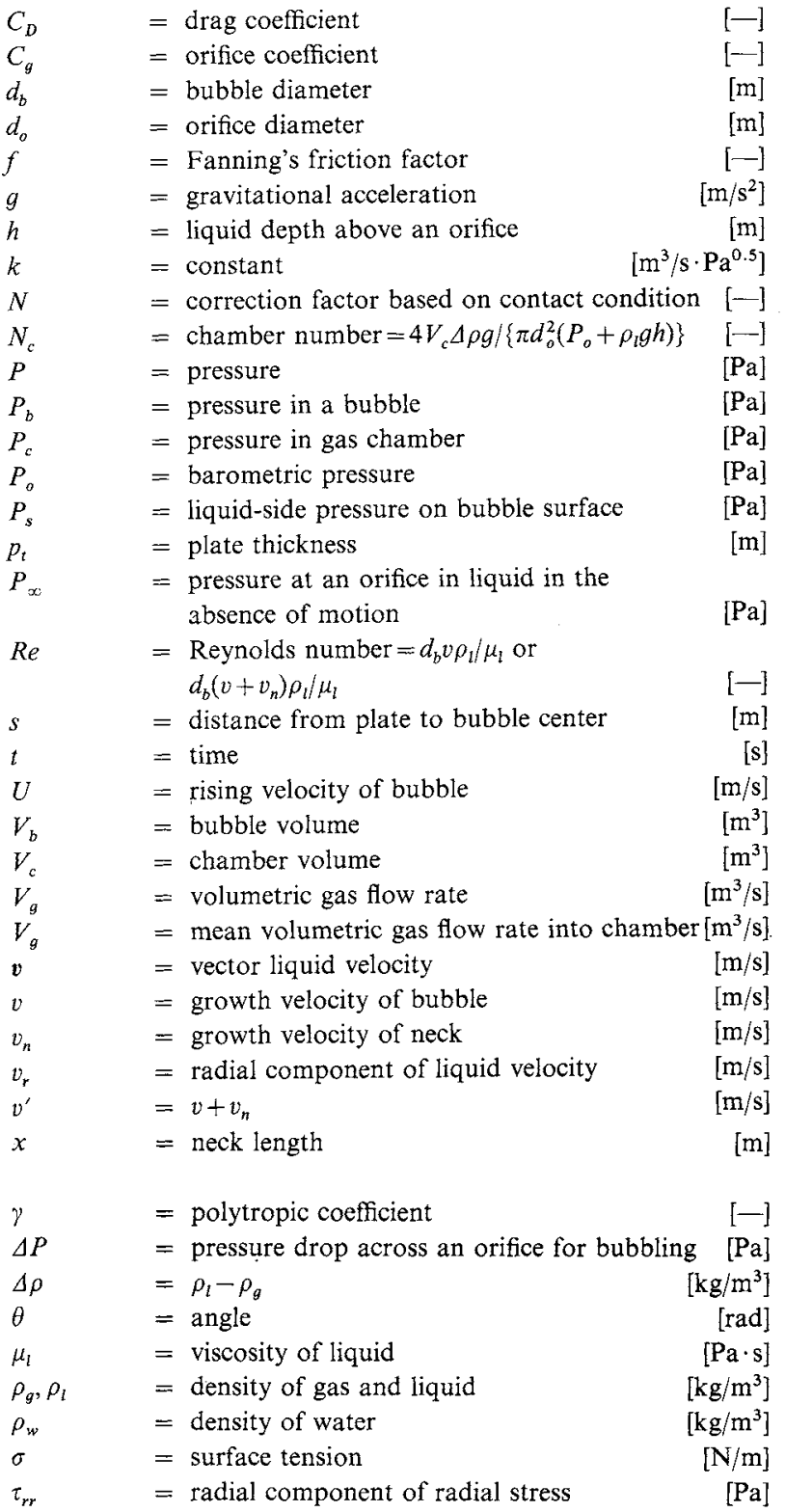

$\langle$ Subscript $\rangle$

$f \quad=$ final value at first stage

\section{Literature Cited}

1) Guy, T. B. and T. J. Jedwidge: Int. J. Heat Mass Transfer, 16, 2393 (1973). 
2) Hadamard, J. S.: Compt. Rend. Acad. Sci., Paris, 152, 1735 (1911).

3) LaNauze, R. D. and I. J. Harris: Chem. Eng. Sci., 27, 2102 (1972).

4) LaNauze, R. D. and I. J. Harris: Chem. Eng. Sci., 29, 1663 (1974).

5) Kumar, R. and N. R. Kuloor: "The Formation of Bubbles and Drops," Advances in Chemical Engineering, 8, 255 (1970).

6) Kupferberg, A. and G. J. Jameson: Trans. Instn. Chem. Engrs., 47, T241 (1969).

7) McCann, D. J. and R. G. H. Prince: Chem. Eng. Sci., 24, 801 (1969).

8) Marmur, A. and E. Rubin: Chem. Eng. Sci., 28, 1455 (1973).

9) Miyahara, T., M. Iwata and T. Takahashi: J. Chem. Eng.
Japan, 17, 592 (1984).

10) Miyahara, T. and T. Takahashi: Kagaku Kogaku Ronbunshu, 9, 592 (1983).

11) Pinczewski, W. V.: Chem. Eng. Sci., 36, 405 (1981).

12) Poritsky, H.: Proc. U. S. First National Congress, Appl. Mech., ASME, 813 (1951).

13) Rybczynski, W.: Bull. Acad. Sci. Cracovie, 1, 40 (1911).

14) Tadaki, T. and S. Maeda: Kagaku Kōgaku, 27, 147 (1963).

15) Takahashi, T. and T. Miyahara: Kagaku Kogaku Ronbunshu, 2, 138 (1976).

16) Takahashi, T. and T. Miyahara: Intern. Chem. Eng., 21, 224 (1981).

17) Tsuge, H. and S. Hibino: J. Chern. Eng. Japan, 11, 173 (1978).

18) Tsuge, H., Y. Tanaka and S. Hibino: Can. J. Chem. Eng., 59, 569 (1981).

\title{
KINETICS OF METHANATION OF CARBON MONOXIDE AND CARBON DIOXIDE
}

\author{
HAKUAI INOUE AND MASAKI FUNAKOSHI \\ Department of Chemical Engineering, University of Tokyo, Tokyo 113
}

Key Words: Chemical Reaction, Chemical Reactor, Methanation, Carbon Monoxide, Carbon Dioxide, Reaction Kinetics, Reaction Mechanism

The kinetics of reaction of carbon monoxide and carbon dioxide with hydrogen in a nickel catalyst tube wall reactor were investigated experimentally and the reaction mechanisms were clarified.

The observed rate laws were

$$
\begin{aligned}
& -r_{\mathrm{CO}}=\frac{k_{1} P_{\mathrm{H}_{2}} P_{\mathrm{CO}}^{1 / 2}}{1+K_{\mathrm{CO}} P_{\mathrm{CO}}} \quad \text { for carbon monoxide, and } \\
& -r_{\mathrm{CO}_{2}}=\frac{k^{\prime} P_{\mathrm{H}_{2}} P_{\mathrm{CO}_{2}}^{1 / 3}}{1+K_{\mathrm{CO}_{2}} P_{\mathrm{CO}_{2}}+K_{\mathrm{H}_{2}} P_{\mathrm{H}_{2}}+K_{\mathrm{H}_{2} \mathrm{O}} P_{\mathrm{H}_{2} \mathrm{O}}}
\end{aligned}
$$

for carbon dioxide at low partial pressure.

These reaction rate laws were derived theoretically from the proposed reaction mechanism. For a mixture of carbon monoxide and carbon dioxide the kinetics of the reaction proved to be well accounted for by the same reaction mechanism.

\section{Introduction}

The methanations of carbon monoxide and carbon dioxide are well-known as industrial catalytic processes for fuel production. ${ }^{8)}$ They have been studied extensively for their industrial applications. Moreover, as standard catalytic reactions their reaction mechanisms have been investigated intensively and the intermediates and reaction steps of methanation were gradually elucidated. . $^{9,10,11}$,

Several kinetic equations for the overall rates of

Received April 6, 1984. Correspondence concerning this article should be addressed to M. Funakoshi. methanation have been proposed, ${ }^{2,4,5)}$ but some of them are too simple to be extended to a wide range of conditions and some are difficult to apply to mixtures of carbon monoxide and carbon dioxide because they ignore the mutual interaction effect on the reaction rates.

In this work the authors studied kinetically the methanation of carbon monoxide and carbon dioxide with a nickel metal catalyst, and tried to derive reasonable and reliable rate equations for the system using a specially devised catalytic reactor, a tube wall catalytic reactor. ${ }^{3)}$

The methanation is exothermic and an increase in 